\title{
Rabies Disease
}

\section{Elsayed Ebrahime*}

Kafrelsheikh University, faculty of veterinary medicine, Egypt

Received: 13 July, 2017; Accepted: 20 July, 2017; Published: 26 July, 2017

*Corresponding author: Elsayed Ebrahime, faculty of veterinary medicine, Kafrelsheikh University, Egypt, Tel: 00201024451052; E-mail: elsayedebrahime@gmail.com

A severe, invariably fetal, viralpolioencephalitis of worm blooded animal including human always fatal following the onset of clinical symptoms. In up to $99 \%$ of cases, domestic dogs are responsible for rabies virus transmission to humans.

Rabies is caused by infection with rabies virus, or other lyssaviruses including Australian bat lyssavirus (ABLV), and is acquired from animals.

Rabies may be transmitted by the bite or scratch of an infected animal virus enter the body through a wound from a bite of rabid animal or via mucous membrane; replicate in myocytes; spread to the neuromuscular junction and neurotendinal spindles travel to Center Nervous System (CNS) via intra-axonal fluid within peripheral nerves; finally spreads centrifugally within peripheral, sensory and motor neurons.

Infection causes tens of thousands of deaths every year, mostly in Asia and Africa. $40 \%$ of people bitten by suspect rabid animals are children under 15 years of age.

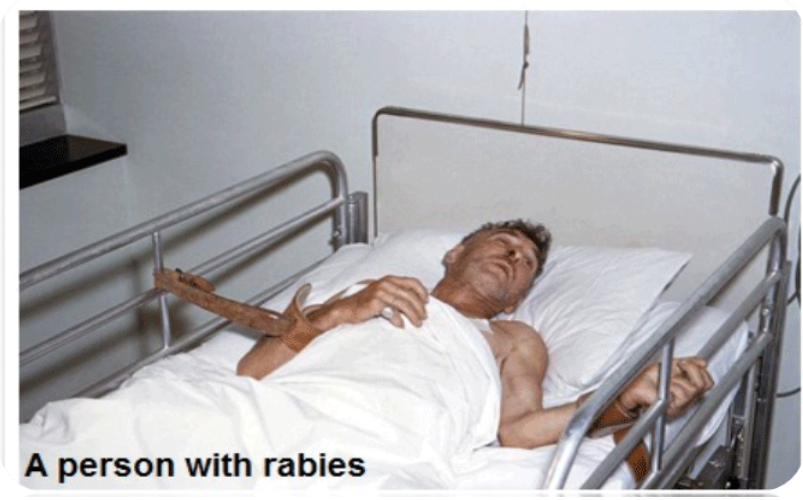

Figure 1: A Person with Rabies

\section{Symptoms of Rabies}

The incubation period for rabies is typically 1-3 months but may vary from 1 week to 1 year, dependent upon factors such as the location of virus entry and viral load ;nearest to CNS is more fatal and fast symptoms appear. Initial symptoms of rabies include a fever with pain and unusual or unexplained tingling, pricking, or burning sensation (paraesthesia) at the wound site. As the virus spreads to the CNS, progressive and fatal inflammation of the brain and spinal cord develops.

Signs and symptoms can include slight or partial paralysis, anxiety, insomnia, confusion, agitation, abnormal behavior, paranoia, terror, and hallucinations, progressing to delirium and coma the person may also have hydrophobia (fear of water). Death usually occurs 2 to 10 days after first symptoms. Survival is rare once symptoms have presented, even with the administration of proper and intensive care.

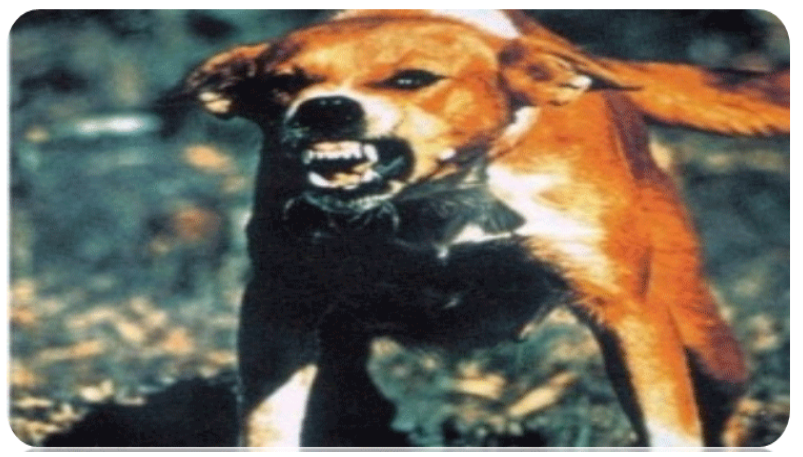

Figure 2:

\section{Diagnosis}

Rabies can be difficult to diagnose, because in the early stages, it is easily confused with other diseases or with aggressiveness.

The diagnosis sample can be taken from saliva, urine and cerebrospinal fluid, but this is not as sensitive and reliable as brain samples

Negri bodies are $100 \%$ diagnostic for rabies infection but are found in only about $80 \%$ of cases. The method for diagnosing rabies is the fluorescent antibody test (FAT).

The FAT relies on the ability of a detector molecule (usually fluorescein isothiocyanate) coupled with a rabiesspecific antibody, forming a conjugate, to bind to and allow the visualization of rabies antigen using fluorescent microscopy 
techniques. Microscopic analysis of samples is the only direct method that allows for the identification of rabies virus-specific antigen in a short time and at a reduced cost, irrespective of geographical origin and status of the host. It has to be regarded as the first step in diagnostic procedures for all laboratories. Autolysed samples can reduce the sensitivity and specificity of the FAT.

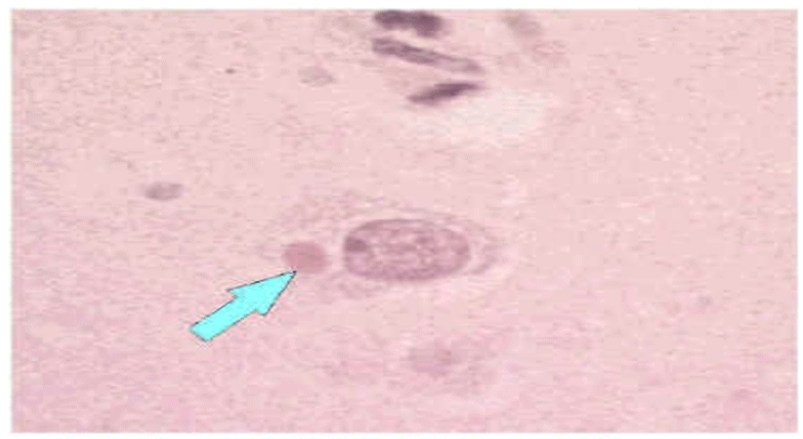

Negri body in body of neuron and positive IFT for rabies antigen

Figure 3: Negri in body of neuon and positive IFT for rabies antigen

\section{Differential Diagnosis}

Must seriously consider rabies for any dog or cat showing unusual mood or behavior changes or exhibiting any unaccountable neurologic signs; caution: handle with considerable care to prevent possible transmission of the virus to individuals caring for or treating the animal.

-Any neurologic disease-brain tumor; viral encephalitis

-Head wound-identify lesions from wound

-Laryngeal paralysis

-Choking

-Pseudo rabies virus infection

\section{Treatment and Vaccination}

Rabies is almost always fatal in domestic animals. Because of the extreme public health danger, treatment is not warranted; therefore, quarantine or euthanize all animals suspected of rabies, and notify local health department authorities.

Rabies prevention requires vaccination and preventing pets from having contact with wild animals

Vaccinate as early as 3 months of age and 1 year later, then booster every 3 years in accordance with the product recommendations. Maternal antibodies from vaccinated females will protect neonates until 3 months of age. Immunity is not fully developed until 28 days after the initial rabies vaccination. Do not vaccinate wild animals or hybrids against rabies, even if they are kept as pets. The safety and efficacy of parenteral rabies vaccination in wildlife and hybrids (wild animal-domestic animal crossbreds) have not been established, and no vaccines are licensed for these animals.

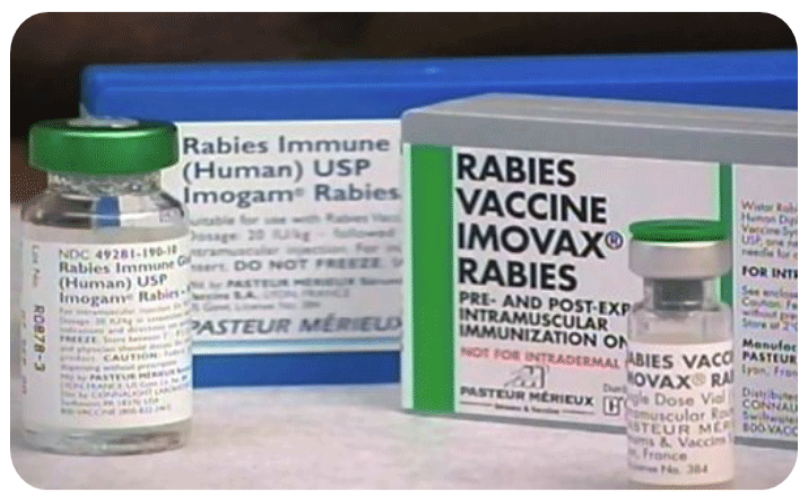

Figure 4:

Jeanna Giese, who in 2004 was the first patient treated with the Milwaukee protocol became the first person ever recorded to have survived rabies without receiving successful post-exposure prophylaxis. 